\title{
BIBLIOTECA DEL HOSPITAL INFANTIL UNIVERSITARIO DE SAN JOSÉ, BOGOTÁ DC.
}

\author{
Jorge Gómez Cusnir MD*
}

Constituye un verdadero honor dirigir estas palabras el día de hoy, con motivo de resaltar la labor y hacer un merecido reconocimiento al profesor Darío Cadena Rey, lo cual en particular es motivo de orgullo y satisfacción para nuestro grupo empresarial de San José y en especial para la Fundación Universitaria de Ciencias de la Salud en este sencillo pero emotivo acto. No es usual en nuestra profesión médica ni tampoco frecuente que lleguen reconocimientos por una gestión o logros alcanzados en el diario trajinar, sin embargo este no es el caso de nuestro querido profesor, pues para la FUCS y su Consejo Superior, queríamos ir más allá de entregar una placa por su vida dedicada a la docencia y a la formación de cientos de estudiantes de pregrado y de patólogos, realizado en la pasada ceremonia solemne de grado de nuestros estudiantes de posgrado de la Facultad de Medicina.

Al rendir este merecido homenaje al profesor Doctor Darío Cadena Rey nuestro actual Vicerrector de Planeación y Proyectos Especiales, miembro de nuestro Consejo Superior y de los órganos de dirección de nuestras instituciones en su calidad de ex-presidente de la Sociedad de Cirugía de Bogotá-Hospital de San José, por su trayectoria y ejecutorias durante más de 40 años en la docencia en calidad de Profesor Titular de la Cátedra de Patología, pero fundamentalmente por sus ejecutorias en todos los cargos directivos que con el paso del tiempo ha ocupado, consiguiendo importantes logros para beneficio de nuestras instituciones.

Quiero reiterarle profesor Darío Cadena mi admiración y aprecio, ha sido un privilegio para mí no sólo haber sido su alumno, sino también haber tenido la fortuna

Presidente Sociedad de Cirugia de Bogotá - Hospital de San José de compartir con usted durante 14 años en que he ejercido el cargo de Presidente de nuestra Sociedad de Cirugía, en la Junta Directiva Hospital de San José y en el Consejo Superior de nuestra institución universitaria, en dónde he tenido la oportunidad de aprender de su experiencia y buen juicio, de su compromiso, dedicación y responsabilidad, con la academia, con la calidad asistencial y con las instituciones que tanto le deben.

Bajo su presidencia y liderazgo el profesor Darío Cadena fué gestor en 1993 de la facultad de Citohistología, única en su género en el país y por iniciativa suya nace en 1998 nuestra propia Facultad de Medicina, con la colaboración del profesor Luis Carlos Taborda su Vicepresidente y el doctor Eduardo Palacios, como Rector de la FUCS para esa época, la cual se constituyó rápidamente en el pilar fundamental para el desarrollo y crecimiento de lo que hoy con orgullo denominamos como Grupo Empresarial de San José y ha sido un soporte financiero invaluable para la buena marcha de nuestros hospitales universitarios.

El sistema de bibliotecas de la Fundación Universitaria de Ciencias de la Salud ha estado ligado al devenir histórico de la fundación misma del Hospital de San José y la Revista Repertorio de Medicina y Cirugía en el siglo pasado. Los miembros de la Sociedad de Cirugía han estado siempre a la vanguardia en la adquisición de colecciones especializadas para el apoyo de la labor asistencial, la academia y la formación de profesionales de la salud en beneficio de nuestra querida patria.

A lo largo de ya más de 111 años, la Biblioteca Arturo Aparicio Jaramillo, llamada así en 1982 en honor del Dr. Aparicio benefactor incansable de las colecciones e información médica, ha funcionado en diferentes lugares del Hospital de San José. En 1990 se asigna el 
espacio que hoy ocupan sus instalaciones con las salas Eduardo Cubides Pardo, Eugenio Ordoñez Márquez y el auditorio Laurentino Muñoz, todos ellos colegas y maestros ilustres de la Sociedad de Cirugía de Bogotá, consagrados al crecimiento y dotación de la biblioteca, la cual ha servido a tantas generaciones de profesionales en ciencias de la salud, desde las primeras escuelas de enfermería, instrumentación quirúrgica y citohistotecnología en el país, de estudiantes de las distintas facultades de medicina que han pasado por nuestros hospitales, los internos y residentes de la Universidad Nacional desde sus inicios hasta 1945, la Universidad Javeriana entre 1946 y 1964, el Colegio Mayor Nuestra Señora del Rosario de 1964 al 2000, de odontólogos, terapistas físicos y respiratorios, auxiliares de enfermería y estudiantes de terapia del leguaje de diferentes universidades y por supuesto hoy en día es el centro de estudio y reunión para los estudiantes de nuestras diferentes Facultades de Medicina, Enfermería, Instrumentación Quirúrgica, Citohistología y de Ciencias Sociales, Administrativas y Económicas.

En 1998 al terminarse el convenio de docencia-servicio entre la Sociedad de Cirugía de Bogotá y la Facultad de Medicina de la Universidad del Rosario, la biblioteca quedó casi desmantelada y su enorme colección de textos se fue con la Universidad del Rosario para la nueva sede de su facultad de medicina en la Quinta Mutis; es para ese entonces que con la guía y dirección del profesor Cadena desde el Consejo Superior, se inicia la política de fortalecimiento de la nueva biblioteca que debía servir las necesidades de actualización y consulta para la comunidad académica de la FUCS y del Hospital de San José.

De esta manera se da la oportunidad de un crecimiento hacia la virtualización y actualización en tecnologías de información y comunicación en la biblioteca, de tal forma que ha sido y sigue siendo soporte fundamental para las actividades asistenciales, académicas, de investigación y proyección social de la Fundación Universitaria de Ciencias de la Salud, del Hospital de San José y del Hospital Infantil Universitario de San José.

Desde el año 2008 cuando la Vicerrectoría de Planeación y Gestión de Proyectos en cabeza del Dr.
Darío Cadena Rey hasta la fecha, el desarrollo y crecimiento de la biblioteca se multiplica de manera exponencial, dándole un especial empuje en su estructura organizacional, conformando el comité de biblioteca como órgano rector de las decisiones del sistema, aprobación y actualización de los reglamentos, implementación de la política de desarrollo de colecciones, así como el aumento en la atención a los usuarios en jornadas extendidas para mayor cobertura y disponibilidad, se amplió en más del $40 \%$ la capacidad de la biblioteca en puestos para lectores y en un buen número de equipos de cómputo y portátiles para uso de los estudiantes.

Así mismo se generó un crecimiento en más del $20 \%$ de material bibliográfico físico y en un $110 \%$ en las colecciones electrónicas disponibles 24 horas, dentro y fuera del campus universitario, con todo tipo de información para el apoyo académico, de investigación y toma de decisión clínica de todos nuestros estudiantes, docentes y administrativos.

El ofrecimiento de nuevos servicios de información como la cátedra electiva en el uso y recuperación de información, bibliochat para la atención en línea de los usuarios y las alertas a traves de mensajes SMS a los móviles de los usuarios,infucs@lud.

El otro gran logro fue la obtención de la certificación de la biblioteca en IS0 9001:2008 convirtiéndose en una de las pocas bibliotecas especializadas certificadas en Colombia y a nivel internacional, y es un ejemplo como pionera para las demás áreas de la institución.

De igual manera la creación y puesta en marcha de convenios y servicios a nivel nacional e internacional como con el Instituto Iberoamericano de Ciencia y Tecnología ISTEC, la Biblioteca Virtual en Salud Colombia, ser centro cooperante del índice más importante de literatura científica y técnica en Salud de América Latina y el Caribe LILACS, y tener actualmente la representación institucional en la presidencia de la Red de Bibliotecas Medicas UNIRECS, son algunos de los logros que debo mencionar de nuestra red de bibliotecas, conseguidos bajo la gestión permanente del profesor Darío Cadena. 
Con la apertura y puesta en marcha de la sede del Hospital Infantil Universitario de San José en el primer semestre de 2009, con más de 60 puestos lector, cubículos de consulta individual, sala grupal y salas digitales dotadas especialmente para el servicio de la comunidad universitaria, que a la fecha atiende a más de 700 usuarios diarios, cada día nuestra biblioteca adquiere mayor importancia por ser el espacio y herramienta permanente para la formación y consulta de nuestros estudiantes y docentes.

Hoy sin duda, somos una de las mejores bibliotecas especializadas en ciencias de la salud que existen en el país y una de las pocas con certificación en normas ISO del ICONTEC desde el finales del año 2011, al servicio de la comunidad FUCS, gracias a liderazgo del Doctor Darío Cadena Rey como Vicerrector de Planeación y Proyectos Especiales y líder del comité de biblioteca de la FUCS que comanda a todo el equi- po de colaboradores de las bibliotecas en cabeza de la bibliotecóloga Marisol Goyeneche Reina, en quién ha recaido la responsabilidad de la dirección operativa de nuestra red de bibliotecas. A todos ellos nuestra gratitud perenne.

Por estos sobrados merecimientos Doctor Cadena, el Consejo Superior de la Fundación Universitaria de Ciencias la Salud y la Rectoría decidieron por unanimidad colocar su nombre a la sede de nuestra biblioteca en el Hospital Infantil Universitario de San José a partir de esta fecha en que se descubre la placa en su honor y reconocimiento. Mis más sinceras felicitaciones para usted Doctor Cadena, para la señora Josefina y sus hijos los doctores María Cecilia, Darío y Marta Lucía Cadena Lleras, pido a ustedes un merecido aplauso,

A ustedes mil gracias. 